\title{
Persée
}

\author{
http://www.persee.fr
}

\section{Protagoras et le panthéon de Thourioi}

\author{
Marco V. García Quintela \\ Revue de l'histoire des religions, Année 2002, Volume 219, Numéro 2 \\ p. $131-139$
}

Voir l'article en ligne

Il y a une profonde similitude entre le panthéon des cités grecques de Téos et d'Abdère, d'une part, et, d'autre part, les dieux dont les noms ont été donnés aux principales avenues de Thourioi. Or Protagoras, législateur à Thourioi, est d'Abdère, et Téos est métropole d'Abdère. On suggère donc que le sophiste a joué un rôle dans l'établissement du panthéon de Thourioi. Ceci implique une collaboration avec d'autres intellectuels présents à Thourioi, comme l'homme politique et spécialiste delphique Lamport ou l'urbaniste et théoricien politique Hippodame. Nous établissons que ces relations sont patentes et connues dans la colonie et nous concluons que cette intervention de Protagoras à Thourioi doit être prise en compte dans l'examen de la théologie du sophiste.

\section{Avertissement}

L'éditeur du site «PERSEE »- le Ministère de la jeunesse, de l'éducation nationale et de la recherche, Direction de l'enseignement supérieur, Sous-direction des bibliothèques et de la documentation - détient la propriété intellectuelle et les droits d'exploitation. A ce titre il est titulaire des droits d'auteur et du droit sui generis du producteur de bases de données sur ce site conformément à la loi nº $98-536$ du 1 er juillet 1998 relative aux bases de données.

Les oeuvres reproduites sur le site «PERSEE » sont protégées par les dispositions générales du Code de la propriété intellectuelle.

Droits et devoirs des utilisateurs

Pour un usage strictement privé, la simple reproduction du contenu de ce site est libre.

Pour un usage scientifique ou pédagogique, à des fins de recherches, d'enseignement ou de communication excluant toute exploitation commerciale, la reproduction et la communication au public du contenu de ce site sont autorisées, sous réserve que celles-ci servent d'illustration, ne soient pas substantielles et ne soient pas expressément limitées (plans ou photographies). La mention Le Ministère de la jeunesse, de l'éducation nationale et de la recherche, Direction de l'enseignement supérieur, Sous-direction des bibliothèques et de la documentation sur chaque reproduction tirée du site est obligatoire ainsi que le nom de la revue et- lorsqu'ils sont indiqués - le nom de l'auteur et la référence du document reproduit.

Toute autre reproduction ou communication au public, intégrale ou substantielle du contenu de ce site, par quelque procédé que ce soit, de l'éditeur original de l'oeuvre, de l'auteur et de ses ayants droit.

La reproduction et l'exploitation des photographies et des plans, y compris à des fins commerciales, doivent être autorisés par l'éditeur du site, Le Ministère de la jeunesse, de l'éducation nationale et de la recherche, Direction de l'enseignement supérieur, Sous-direction des bibliothèques et de la documentation (voir http://www.sup.adc.education.fr/bib/ ). La source et les crédits devront toujours être mentionnés. 


\author{
MARCO V. GARCÍA QUINTELA
}

Université de Saint-Jacques-de-Compostelle

\title{
Protagoras et le panthéon de Thourioi
}

Il y a une profonde similitude entre le panthéon des cités grecques de Téos et d'Abdère, d'une part, et, d'autre part, les dieux dont les noms ont été donnés aux principales avenues de Thourioi. Or Protagoras, législateur à Thourioi, est d'Abdère, et Téos est métropole d'Abdère. On suggère donc que le sophiste a joué un rôle dans l'établissement du panthéon de Thourioi. Ceci implique une collaboration avec d'autres intellectuels présents à Thourioi, comme l'homme politique et spécialiste delphique Lampon ou l'urbaniste et théoricien politique Hippodame. Nous établissons que ces relations sont patentes et connues dans la colonie et nous concluons que cette intervention de Protagoras à Thourioi doit être prise en compte dans l'examen de la théologie du sophiste.

\section{Protagoras and the Tourian pantheon}

There is a profound analogy between the gods worshipped at the ancient Greek cities of Teos and Abdera, its metropolis, and the gods after which the Thourioi's avenues were named. As Protagoras the Sophist was born in Abdera and he established the laws of Thourioi, we may thus think of our Sophist as the mentor of the Thourian pantheon. This would imply a cooperative action between Protagoras and some intellectuals such as the Delphic seer Lampon and the urbanist Hippodamos, both also present at the foundation of Thourioi. Finally, it seems very likely that the links among de pantheons of the above cited cities were well known, and so we could conclude that Protagoras' action at Thourioi should be considered in the studies on the religious thought of this Sophist. 
Protagoras est, sans aucun doute, un cas exemplaire d'individu à la pensée complexe. Il était athée ou du moins agnostique'. Mais Platon lui attribue une version particulière du mythe de Prométhée qui présente Zeus comme le fondateur et le garant de l'ordre social tel qu'on le comprenait dans la vie des cités grecques. Homme de pensée, il décrit son activité de sophiste comme l'enseignement du principe homérique et aristocratique consistant à lier la parole à l'action, la parole dans le conseil à l'action courageuse du guerrier'2. Mais, à cause de sa condition juridique d'étranger dans les villes où il a résidé presque toute sa vie, il n'a jamais pu mettre en œuvre cet idéal ${ }^{3}$.

Mais il y a peut-être un moment de sa vie où ces deux paradoxes s'entrecroisent et se résolvent d'une façon particulière. Il s'agit de sa participation à la fondation de la colonie panhellénique de Thourioi en l'an 444 av. J.-C.

Nous possédons seulement un témoignage de la présence de Protagoras à Thourioi en tant que législateur ${ }^{4}$. Diodore, notre source principale sur Thourioi, n'y fait pas référence aux deux occasions qui sembleraient appropriées. D'une part, quand il explique l'organisation tribale de Thourioi et sa constitution démocratique; d'autre part, quand il

1. Il a peut-être été persécuté pour cela, voir A. B. Drachman, Atheism in Pagan Antiquity, Chicago, Ares, 1977 (Londres, 1922), p. 39-42 ; W. K. C. Guthrie, Historia de la Filosofia Griega, vol. III, Siglo v. Ilustración, Madrid, Gredos, 1988, p. 231-233. Sa mise en accusation est discutée : cf. E. Derenne, Les procès d'impiété intentés aux philosophes à Athènes au $V^{*}$ et au IV siècle avant J.-C., Liège-Paris, 1930, p. 44-55; bibliographie sur ces deux aspects dans M. Winiarczyk, Bibliographie zum antiken Atheismus 17. Jahrhundert-1990, Bonn, 1994, p. 83-85, définition religieuse et philosophique, et 105-106, accusation.

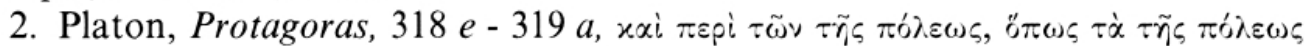

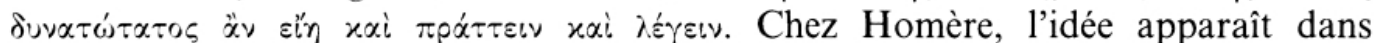
L'Iliade, I, 258; II, 202; IX, 443. Dans la tradition philosophique Héraclite l'emploie fréquemment, B 1, B 29, B 73, B 74, B 112, commentés dans M. V. García Quintela, El rey melancólico. Antropologia de los fragmentos de Heráclito, Madrid, 1992, p. 105-113; cf. Démocrite, B 2.

3. J. A. Davidson, "Protagoras, Democritus and Anaxagoras", Classical Quarterly, n.s., 3, 1953, p. 33-45: ses sources sont discutables, mais il est préférable à J. S. Morrison, "The Place of Protagoras in Athenian Public Life ", Classical Quarterly, 35, 1941, p. 1-16 ; cf. G. B. Kerferd, The Sophistic Movement, Cambridge, 1981, p. 48.

4. Héraclide du Pont, Sur les lois, fragment $150 \mathrm{Wehrli}$, = Diogène Laërce IX, 8, 50. N. O'Sullivan, «Pericles and Protagoras", Greece \& Rome, 42, 1995, p. 1523, surtout p. 17-18, a suggéré que les Aoupropdìveıı cités par Aristophane, Nuées, 331-332, font allusion au sophiste. 
attribue à Charondas la responsabilité de la législation de la cité. Cette carence s'allie à l'absence dans le texte de Diodore des autres intellectuels présents à Thourioi (Hippodame, Hérodote, Lysias, Empédocle, etc.), lesquels sont connus grâce à d'autres témoignages : le Sicilien cite seulement les Athéniens Lampon et Xénocritos. Mais plus loin Diodore fait allusion, sans les citer, à tous ceux-ci au sujet de la querelle concernant le fondateur qui s'est produite à Thourioi, quand il dit :

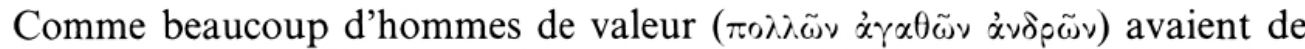
même concouru à cette entreprise et y avaient été très utiles, la discussion allait bon train, car chacun brûlait d'obtenir ce titre de fondateur?

On a résolu la querelle en recourant à l'oracle de Delphes qui a attribué la fondation à Apollon lui-même (c'était en l'an 434 av. J.-C.). En partant de cette situation, il est possible de suggérer une hypothèse. L'absence d'Hippodame, Protagoras, Hérodote, etc., à la fondation de Thourioi telle que l'explique Diodore reflète le consensus civique atteint après la querelle sur le fondateur. À partir de ce moment, il serait de mauvais goût dans la cité d'insister sur les services de tant "d'hommes de valeur". Ainsi s'explique le fait que Lampon, le devin delphique proche d'Apollon, soit le seul expressément cité avec Xénocritos?. Timée, qui écrit à Athènes, ou ses sources, que Diodore suit, se feraient l'écho de cette doctrine officielle (thourio-delphique) sur l'oikiste de Thourioi, ce qui pourrait expliquer que les célébrités qui ont participé à la création de la cité ne sont pas mentionnées ${ }^{8}$. Le silence de Diodore au sujet du sophiste nous semble donc explicable.

5. Diodore, XII, 11, 3-4. Cf. D. Plácido, "Protágoras y Pericles », Hispania Antiqua, 2, 1972, p. 7-19, surtout p. 12-18; l'attribution est invraisemblable vu le caractère légendaire du personnage: A. Delatte, Essai sur la politique pythagoricienne, Genève, 1979 (Paris-Liège, 1922), p. 177-188.

6. Diodore XII, 35, 3. Texte et trad. M. Casevitz, Diodore de Sicile. Bibliothèque historique, livre XII, Paris, 1972. Casevitz omet $\alpha \gamma \alpha \theta \tilde{\omega} v$ sans le justifier, mais traduit "beaucoup d'hommes de valeur". C. H. Oldfather, Diodorus of Sicily IV,

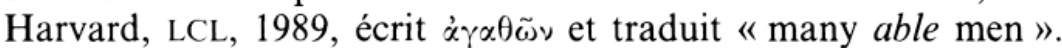

7. Lampon : I. Malkin, Religion and Colonization in Ancient Greece, Brill, Leiden, etc., 1987, p. 97-101 ; Xénocrite : M. Moggi, "Senocrito, Tucidide di Melesia e la fondazione di Turi (Anonym., Vit. Thuc., 6-7) ", Annali Scuola Normale Superiore Pisa, III, 9/2, 1979, p. 499-504.

8. Hippodame à Thourioi apparaît chez un lexicographe et dans une scolie, D.-K. 39 A 3 et 4. Protagoras, supra, n. 4. Hérodote chez Cornelius Nepos cité par Pline, NH, XII, 18 ; Strabon, XIV, 2, 16 ; Julien, Epist., 22 ; Souda s.v. Herodotos; voir D. Asheri, Erodoto, Le Storie. Libro I, La Lidia e la Persia, Milan, 1988 , p. 261. À l'encontre de la communis opinio, N. K. Rutter, "Diodorus and the Foundation of Thurii ", Historia, 22, 1973, p. 155-176, considère qu'Éphore est la source de Diodore, p. 161, contra L. Pearson, "Ephorus and Timaeus in Diodorus ", Historia, 33, 1984, 1-20. 
L'identification du rôle que Protagoras a pu jouer dans la colonie est un autre problème. Dans deux articles classiques, J. S. Morrison et $\mathrm{V}$. Ehrenberg ${ }^{9}$ le considèrent comme fondamental. Grâce à une analyse plus détaillée, V. Muir a trouvé dans la loi de Thourioi qui oblige à apprendre à lire aux enfants le reflet d'un précepte de Protagoras, que l'on peut attribuer aussi à Hippodame ${ }^{10}$. Dans d'autres études, la question n'est même pas posée. C'est le cas par exemple pour le livre important de C. Farrar, lequel s'intéresse au rôle fondamental joué par Protagoras dans la fondation de l'idéologie démocratique, mais ne fait aucune mention de son intervention politique réelle à Thourioi ${ }^{11}$. D'autres travaux réunis par G. B. Kerferd portant sur divers aspects de Protagoras en tant qu'homme politique, mais dans le cadre de l'histoire de la philosophie, ignorent aussi cette question $^{12}$.

Il me semble qu'aucun des deux types d'études citées ci-dessus ne pose correctement les termes de la relation qui peut exister. Ainsi Morrison et Ehrenberg, partant de l'histoire politique traditionnelle, ne considèrent Protagoras que comme un homme de parti, et le présentent comme un mentor dans l'ombre de Périclès ou comme un continuateur de l'initiative de ce dernier. Alors que Farrar et les autres, occupés à établir la place du sophiste dans la dialectique des idées, oublient qu'il s'agit d'un personnage réel, discernable et actif.

J'envisage d'analyser dans un autre travail la relation profonde, structurelle, qui existe entre le système de tribus établi à Thourioi et le mythe de Prométhée tel qu'il apparaît dans le Protagoras. Ici, je me bornerai à signaler une coïncidence formelle frappante entre les panthéons des cités de Thourioi, Abdère, patrie du sophiste, et Téos, métropole d'Abdère.

9. J. S. Morrison, art. cité ; V. Ehrenberg, "The Foundation of Thurii ", American Journal of Philology, 69, 1948, p. 149-170, surtout p. 168-169.

10. J. V. Muir, "Protagoras and Education at Thourioi ", Greece \& Rome, 39, 1982, p. 17-24, et M. V. García Quintela, «Hipódamo en Turios : urbanismo, religión y política ", Dialogues d'Histoire Ancienne, 26/1, 2000, p. 9-35, voir p. 34-35.

11. C. Farrar, The Origins of Democratic Thinking, Cambridge, 1988, p. 45, n. 5, suit K. J. Dover quand il considère que le témoignage d'Héraclide du Pont " cannot be regarded as firm evidence". Il y a des années, D. Loenen se passait de Thourioi, et même d'Athènes! (D. Loenen, Protagoras and the Greek Community, Amsterdam, 1940, p. 128).

12. Ch. H. Kahn, "The Origins of Social Contract Theory", p. 98-108; K. Döring, "Die politische Theorie des Protagoras ", p. 109-115 ; T. J. Saunders, "Protagoras and Plato on Punishment", p. 129-141 ; dans G. B. Kerferd (ed.), The Sophists and Their Legacy, Wiesbaden, 1981. 
En ce qui concerne Thourioi, Diodore présente ainsi le tracé urbain de la cité et les noms de dieux donnés aux rues:

Ils divisèrent ensuite la ville en quatre avenues dans le sens de la longueur, appelant l'une avenue d'Héraclès, l'autre d'Aphrodite, la troisième d'Olympie, la quatrième de Dionysos; dans le sens de la largeur, ils la divisèrent en trois avenues, appelées l'une Hérôa, l'autre Thouria, la troisième Thourina. Quand ils eurent rempli de maisons les petites rues, la cité parut parfaitement organisée (XII, 10, 7 ; texte et trad. M. Casevitz).

Le texte présente des difficultés philologiques analysées par des historiens de l'urbanisme ${ }^{13}$ et le problème de sa relation avec l'archéologie de la cité ${ }^{14}$ se pose. Mais il est significatif que ce que dit Diodore concorde, dans des limites évidentes, avec ce que les archéologues ont identifié sur le terrain.

Ce qui nous intéresse maintenant c'est d'établir que pour la fondation des cités grecques divers spécialistes, plus concrètement les devins et les architectes, travaillent à l'unisson, chacun jouant son rôle spécifique dans un but commun. On peut le voir, par exemple, à Colophon à la fin $\mathrm{du} \mathrm{IV}^{\mathrm{e}}$ siècle av. J.-C. : un texte épigraphique signale l'action des spécialistes religieux présidés par un prêtre d'Apollon, comme Lampon à Thourioi, et un peu plus bas l'intervention des architectes dans la fondation ${ }^{15}$, comme Hippodame à Thourioi. D'autre part, lors de la refondation de Messène après la bataille de Leuctres en 371

13. G. Vallet, "Avenues, quartiers et tribus à Thourioi, ou comment compter les cases d'un damier (à propos de Diod. XII, 10 et 11)", in Mélanges Heurgon, vol. II, Rome/Paris, 1976, p. 1021-1033. F. Castagnoli, Orthogonal Town Planning in Antiquity, Cambridge (Mass.), 1971, p. 32-35.

14. G. Guzzo, "Sibari e la Sibaritide : materiali per un bilancio della conoscenza archeologica ", Revue archéologique, 1992/1, p. 3-35, sur Thourioi, p. 20-25, et, presque le même texte dans P. L. Guzzo, "Sibari. Materiali per un bilancio archeologico ", dans Sibari e la Sibaritide, Taranto, 1992, p. 51-82, p. 66-70 sur Thourioi, et E. Greco, "Turi ", dans E. Greco (éd.), La città greca antica. Istituzioni, società e forme urbane, Rome, 1999, p. 413-430, voir p. 416-419. Sur la topographie et la géologie du territoire, voir A. D’Arrigo, Premessa geofisica alla ricerca di Sibari, Naples, 1959, passim, et V. Cotecchia, "Incidenze geologiche e geotecniche su Sibari e la Sibaritide ", dans Sibari e la Sibaritide, Taranto, 1992, p. 21-49. Cf. www.perseus.tufts.edu/cgi-bin/text?lookup=pecs +thurii et www.perseus.tufts.edu/cgi-bin/text?lookup=pecs+sybaris avec des photographies des fouilles de 1992.

15. Texte de l'inscription dans B. D. Meritt, "Inscriptions of Colophon ", American Journal of Philology, 56, 1935, p. 358-395, $\mathrm{n}^{\circ} \mathrm{I}$, p. 359-372, lignes 12-13 sur les prêtres, lignes 15-18 sur les cultes, étudiées par G. Sissa, M. Detienne, $L a$ vie quotidienne des dieux grecs, Paris, 1989, p. 202-203 ; lignes 23-28 pour les architectes et les tâches dont ils ont la charge, étudiées par R. Martin, L'Urbanisme dans la Grèce antique, Paris, 1974, p. 55-56 pour les aspects urbanistiques. Au sujet de Lampon, voir supra, n. 9. 
av. J.-C., on fait des sacrifices aux dieux des cités qui aident à la reconstruction, Thèbes et Argos, mais surtout aux divinités et héros locaux dont on sollicite la protection pour la nouvelle fondation. Des architectes sont également cités dans le récit de Pausanias sur cette fondation ${ }^{16}$.

Il n'est donc pas étonnant que le panthéon de Thourioi se compose de divinités épichoriques, pour les voies orientées est-ouest, et de divinités panhelléniques pour celles orientées nord-sud, situation identique à celle de la cité dont Platon décrit la fondation dans Les Lois ${ }^{17}$. Ce qui est propre à Thourioi c'est que le tracé urbain et le panthéon s'identifient. Ils ne sont pas présentés comme le résultat de sphères d'activité différenciées, comme c'est le cas dans les témoignages sur Colophon ou Messène, où le rôle concret des divers intellectuels impliqués dans la fondation reste flou.

C'est pourquoi les cas présentant des analogies avec le panthéon de Thourioi sont éclairants. En effet, ce sont les mêmes dieux qui président aux fêtes publiques dans les cités d'Abdère et de Téos. Le témoignage relatif à Téos est un épigraphe daté d'environ 470 av. J.-C. qui

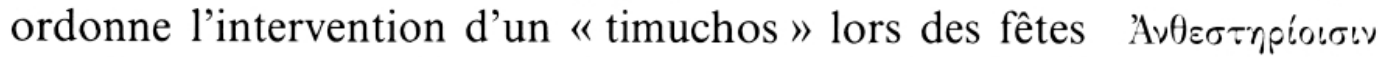

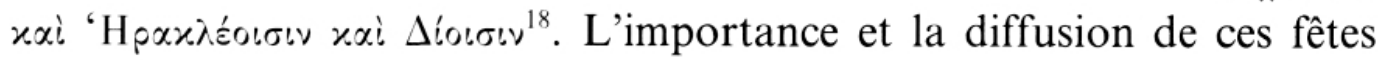
sont confirmées par la publication en 1981 d'un autre épigraphe de Téos qui révèle qu'elles sont aussi en vigueur dans sa colonie

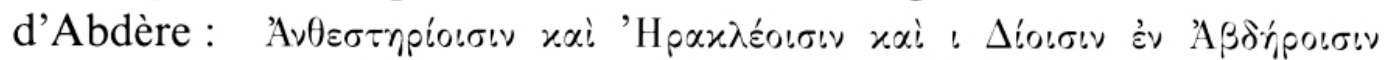

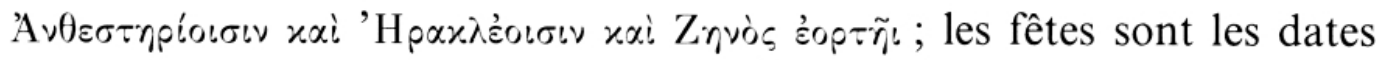
prescrites pour lire le contenu de l'épigraphe en question ${ }^{19}$.

Si nous pensons aux dieux en l'honneur desquels on célèbre les fêtes dans les deux cités de l'Égée, nous trouvons tout d'abord Dionysos, présent aux Anthestéries, puis Héraclès et Zeus, probablement

16. Pausanias, IV, 26, 5 - 27, 8, commenté par Sissa, Detienne, op. cit., p. 203205 ; I. Malkin, op. cit., p. 104-106. Cf. I. Malkin, "What were the Sacred Precincts of Brea (IG I $\left.{ }^{3}, \mathrm{n}^{\circ} 46\right)$ ", Chiron, 14, 1984, p. 43-48, situation similaire à Brea et Abdère, p. 47-48. Pausanias, IV, 27, 5, fait état de la coopération entre devins et urbanistes.

17. Platon, Les Lois, V, 745 b, cite Hestia, Zeus et Athena ; et VIII, $848 d$,

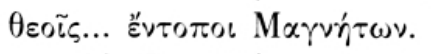

18. R. Meiggs, D. Lewis, A Selection of Greek Historical Inscriptions, Oxford, 1989, n $^{\circ} 64$, p. $62-66$.

19. P. Herrmann, "Teos und Abdera im 5. Jahrhundert v. Chr. Ein neues Fragment der Teiorum Dirae ", Chiron, 11, 1981, p. 1-30, réétudié par A. J. Graham, "Abdera and Teos ", Journal of Hellenic Studies, 112, 1992, p. 44-73, surtout p. 55-59. 
avec l'épithète de Meilichios, en relation avec les Diasia ${ }^{20}$. Comme on peut le voir, seule manque Aphrodite pour que l'on ait le même panthéon qu'à Thourioi. D'autre part, le lien entre Thourioi et Abdère se produit nécessairement à travers Protagoras qui, selon le poète comique Eupolis, son contemporain, se présente aussi comme Téien ${ }^{21}$. Ce qui est conforme à l'usage fréquent selon lequel les citoyens des colonies sont considérés comme citoyens appartenant aussi aux métropoles.

Il y aurait donc lieu de voir dans le sophiste un homme soucieux de la formation du panthéon de Thourioi et apportant concrètement les dieux de sa terre natale comme structure de base du panthéon de la nouvelle cité $^{22}$. Malheureusement, le système des fêtes de Téos et Abdère ne présente pas d'éléments permettant de mieux comprendre le sens de la présence de ces divinités à Thourioi. On peut peut-être affirmer qu'à Thourioi les dieux ont une dimension olympienne, établie par l'allusion à Zeus "d'Olympie", tandis que la mention des Diasia à Téos et à Abdère renvoie à Zeus Meilichios, avec un aspect chthonien très présent, aussi, dans les Anthestéries en l'honneur de Dionysos, et l'on connaît Héraclès, parmi tant d'autres choses, à cause de sa descente aux Enfers ${ }^{23}$.

Une autre inscription d'Abdère récemment publiée montre que le lien entre les panthéons de Thourioi et d'Abdère peut aussi apparaître dans les avenues qui portent des noms de divinités épichoriques. Comme nous l'avons vu l'une d'entre elles est dédiée aux héros alors

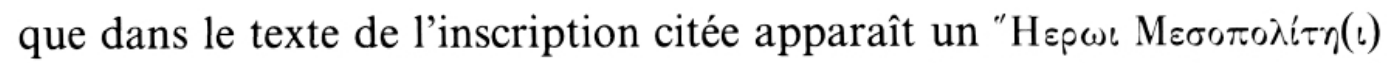
que les éditeurs relient à Abdère, l'éromènos d'Héraclès qui a donné

20. Au sujet de la fête, J. Harrison, Prolegomena to the Study of Greek Religion, Londres, 1962, p. 12-28; A. B. Cook, Zeus. A Study in Ancient Religion, vol. II, Zeus god of the Dark Sky, Part. II : Appendixes and index, New York, 1965, p. 1091-1160, et le matériel compilé par E. Mitropoulou, Deities and Heroes in the Form of Snakes, Athènes, 1977, p. 112-155.

21. Eupolis, fragment 157 Kassel-Austin (= Diogène Laërce, IX, 50). Il en est de même chez l'historien Hécatée, originaire d'Abdère ou de Téos: voir Ps.Scimno dans Strabon, FrGrHist $264 \mathrm{~T}$ 1, et pour cette affinité entre les deux cités, voir G. B. D'Alesio, "Immigranti a Teo e ad Abdera (SEG XXXI 985, Pind. fr. 25 B Sn.-M.) ", Zeitschrift fur Papyrologie und Epigraphik, 92, 1992, p. 73-80, p. 77, et passim, et voir supra, n. 19.

22. Concordance avec I. S. Mark, "The Gods on the East Frieze of the Parthenon ", Hesperia, 53/3, 1984, 289-342, qui, aux p. 317-330, compare les dieux et les idées du mythe de Prométhée du Protagoras de Platon avec le panthéon du Parthénon.

23. Analyse du panthéon de Thourioi dans García Quintela, « Hipódamo en Turios ". 
son nom à la cité lors de sa première fondation mythique ${ }^{24}$. Dans ce cas, le parallélisme avec Thourioi est double: d'une part, l'avenue Hérôa est mésopolites dans la fondation italique, à cause de sa localisation; d'autre part, aux deux endroits on fait une différence entre Héraclès, qui fait partie des dieux, et les Héros, auxquels on rend un culte apparemment distinct.

Il convient d'ajouter une considération aux faits exposés. La relation entre ces panthéons ne devait pas poser de problème car on devait disposer à Thourioi de nombreuses informations au sujet des cités de l'Égée. Hérodote, qui est allé aussi à Thourioi relate la colonisation d'Abdère par les Téiens ( $I, 168)$ et la proposition de Thalès selon laquelle Téos devrait occuper une place centrale dans une Ionie confédérée (I, 170,3). De plus, l'une des dix tribus de Thourioi s'appelait Ionie (Diodore, XII, 11,3) et pouvait comporter des colons des cités d'Asie Mineure et de leurs colonies au courant de ces questions locales, sans exclure, bien entendu, des personnes originaires de Téos et Abdère comme Protagoras. Cette tribu pourrait inclure, par exemple, Hippodame de Milet. Hérodote évoque aussi le projet de Bias de Priène concernant la colonisation panionienne de la Sardaigne (I, 171, 1-2) et Thourioi est très proche.

Il s'agit en tout cas de l'adaptation d'un itinéraire bien connu qui allait d'est en ouest. C'est celui des Phocéens qui fondent Alalia en Sardaigne puis Élée. Le même itinéraire revient à l'esprit des Grecs vainqueurs à Platée lorsqu'ils cherchent un endroit acceptable pour les Ioniens ${ }^{25}$. C'est, aussi, le choix des aristocrates de Samos qui, fuyant la tyrannie de Polycrate, fondent Dikearchia dans le Golfe de Naples puis, après la révolte ionienne, s'installent à Zancle ${ }^{26}$. C'est aussi l'itinéraire des philosophes, du Dorien Épicharme de Cos installé dans la Syracuse dorienne (Diogène Laërce, VIII, $78=$ DK 23 A 3, cf. A 1,

24. D. Kallintz, Ch. Veligianni, "Eine neue Weihinschrift aus Abdera", Ancient History Bulletin, 10, 1996, p. 51-65. Les éditeurs datent l'inscription du dernier tiers du IV $V^{e}$ siècle av. J.-C., p. 55, mais si leur analyse religieuse est correcte, p. 59-62, il est probable que le culte reflété est beaucoup plus ancien.

25. Hérodote, I, 163-167 et IX, 106, 2, et cf. VI, 17 sur Dionysos de Phocée, corsaire contre les Phéniciens entre l'Italie et la Sicile après la défaite des Ioniens à la fin du $\mathrm{VI}^{\mathrm{e}}$ siècle. Au sujet du sens de ces mesures et de leur valeur chez Hérodote, voir N. Demand, "Herodotus and Metoikesis in the Persian Wars ", American Journal of Philology, 109/3, 1988, p. 416-423, sur Téos, p. 418.

26. Eusèbe (St. Jerôme), Ol. 62 [circa 529], Samii Dicaearchiam condiderunt quam nunc Poteolos uocant, et J. P. Barron, "The Sixth-century Tyranny at Samos ", Classical Quarterly, ns. 14/2, 1964, p. 210-229, p. 228 et n. 2, et Hérodote, VI, 22. 
avec d'autres origines), de l'Ionien Xénophane de Colophon installé à Élée l'Ionienne ${ }^{27}$, de l'Ionien Pythagore de Samos installé à Crotone l'achéenne ${ }^{28}$. D'autre part, l'archéologie détecte de nombreux restes d'amphores provenant de cités grecques d'Orient dans beaucoup de gisements siciliens; il s'agit donc aussi de routes commerciales ${ }^{29}$. Et c'est, enfin, l'itinéraire de Protagoras, Hérodote et Hippodame qui finissent à Thourioi après une escale à Athènes.

Toutes ces données permettent de discerner une relation ou une collaboration entre les intellectuels qui apparaissent à Thourioi, au moins à l'origine. Lampon, connaisseur des traditions religieuses helléniques en général et athéniennes en particulier, est aussi sensible aux traditions d'un endroit déjà hellénisé qui conserve le souvenir des puissances épichoriques et des traditions achéennes. Hippodame, architecte, urbaniste et porteur d'un projet politico-social particulier, définit un tracé quadrangulaire et idéalement expansif dans l'espace (en concordance avec le caractère ouvert de la citoyenneté de Thourioi, qui accepte tous les Grecs), lequel s'oppose au tracé circulaire, avec un nombre limité de citoyens, défini par Platon dans Les Lois. Enfin, Protagoras apporte avec lui le panthéon de sa patrie auquel il a su donner un vernis panhellénique dans le contexte de la nouvelle fondation : il agit alors comme beaucoup d'oikistes grecs ${ }^{30}$. Ainsi, lorsqu'il a l'occasion d'unir la parole à l'action, de réaliser sur le plan historicomatériel le monde de ses idées, le sophiste est plus près de la tradition que de l'innovation, au moins en matière religieuse. Il convient donc d'avoir cette situation présente à l'esprit lorsque l'on étudie les opinions théologiques de Protagoras qui peuvent être déduites du contenu des fragments et témoignages conservés.

Departamento de Historia 1

Facultade de Xeografía e Historia

E-15703 Santiago de Compostela

27. Diogène Laërce, IX, 18 et $20(=\mathrm{DK} 21$ A 1$)$. Clément, Str., I, 64 (DK $21 \mathrm{~A} \mathrm{8}$ ), en fait le fondateur de l'école d'Élée. M. Gigante, "Senofane e "la colonizzazione di Elea" ", Parole del Passato, 20, 1970, p. 236-240, rejette l'information selon laquelle Xénophane aurait composé une Colonisation d'Élée.

28. Jamblique, $V P, 11$, cf. 88 ; Porphyre, $V P, 7,9(=16)$. Pour le contexte, voir G. Shipley, A History of Samos 800-188 BC, Oxford, 1987, p. 91, cf. p. 72-91.

29. R. M. Albanese Procelli, «Appunti sulla distribuzione delle anfore com-

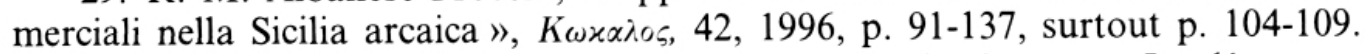

30. I. Malkin, «La place des dieux dans la cité des hommes. Le découpage des aires sacrées dans les colonies grecques ", Revue de l'histoire des religions, 204, 1987 , p. $331-352$. 